\title{
Eficacia de los tests de Helkimo y Krogh - Paulsen en el diagnóstico de la disfunción tempormandibular
}

\section{Effectiveness of the Helkimo and Krogh-Paulsen tests in the diagnosis of tempormandibular dysfunction}

\author{
Emilio, Gomez
}

\section{RESUMEN}

Objetivo: el presente estudio se planteó para evaluar la eficacia de los Test de Helkimo y Krogh - Paulsen para el diagnóstico de la disfunción temporomadibular, además de determinar por medio de las pruebas de sensibilidad y especificidad la consistencia para el Test Krogh - Paulsen. Materiales y Métodos: se seleccionaron 30 sujetos con edades comprendidas entre $15 \pm 30$ años inscritos en la Escuela de Estomatología de la Universidad Alas del Perú, los cuales fueron examinados por medio de los Test de Helkimo y Krogh - Paulsen de acuerdo a los indicadores de cada instrumento, estos fueron evaluados clínicamente con la instrumentaría adecuada para diagnosticar disfunción temporomadibular. Los datos fueron analizados por pruebas estadísticas apropiadas con un valor de $p<0,05$. Resultados: El Test Helkimo presenta resultados positivos de disfunsión temporomadibular en un 93,3\% y el Test Krogh - Paulsen 76,6\%, aún cuando la prueba $\mathrm{Chi}=3,268$ con un $\mathrm{p}=0,0706$ lo cual muestra que no hay diferencias significativas entre ambos test. Posteriormente se efectuó la prueba de sensibilidad con un 78,6\% y de especificidad con 50\%. Conclusiones: El Test de Helkimo y Krogh - Paulsen no presentan diferencias en su capacidad de diagnostico, enfatizando la congruencia metodológica en cuanto a comparación de instrumentos para el diagnostico de la disfunción temporomadibular, además del establecimiento de parámetros metodológicos para las interpretaciones de dichos test.

Palabras claves: Disfunción Temporomandibular, Test de Helkimo, Test de Krogh - Paulsen.

\section{ABSTRACT}

Objective: the present study was proposed to evaluate the efficacy of the Helkimo and Krogh-Paulsen tests for the diagnosis of temporomadibular dysfunction, in addition to determining the consistency for the Krogh-Paulsen Test by means of sensitivity and specificity tests. Materials and Methods: 30 subjects with ages between $15 \pm 30$ years enrolled in the School of Stomatology of the Universidad Alas del Perú were selected, which were examined by means of the Helkimo and Krogh-Paulsen tests according to the indicators of each instrument, these were clinically evaluated with the appropriate instruments to diagnose temporomadibular dysfunction. The data were analyzed by appropriate statistical tests with a value of $\mathrm{p}<0.05$. Results: The Helkimo Test presents positive results for temporomadibular dysfunction in $93.3 \%$ and the Krogh - Paulsen Test $76.6 \%$, even when the Chi $=3.268$ test with a $p=0.0706$ which shows that there are no significant differences between both tests. Subsequently, the sensitivity test was carried out with $78.6 \%$ and the specificity with 50\%. Conclusions: The Helkimo and Krogh - Paulsen test do not present differences in their diagnostic capacity, emphasizing the methodological congruence regarding the comparison of instruments for the diagnosis of temporomadibular dysfunction, in addition to the establishment of methodological parameters for the interpretations of said tests.

Key words: Temporomandibular dysfunction, Helkimo test, Krogh-Paulsen test 


\section{INTRODUCCIÓN}

A nivel mundial la mayor causa de la consulta odontológica se debe a la caries dental y a enfermedades periodontales, y solo cuando estas presentan algún grado de dolor o de impedimento para las actividades diarias del paciente, lo mismo ocurre cuando de afecciones sobre el aparato gnático se trata, aún cuando más de $70 \%$ de la población mundial (1) padezca de este tipo de afecciones, la principal causa de asistencia a la consulta odontológica es la presencia de dolor incapacitante en este sistema (2), este tipo de sintomatología presenta relativa importancia en la calidad de vida del individuo ya que se encarga de las acciones de la masticación, deglución, fonación y expresividad facial (1).

Este tipo de sintomatologías son clasificadas como disfunción o trastorno en la Articulación Temporomandibular (TTM), las cuales van desde ruidos en la articulación, dolores de los músculos masticadores, alteraciones en el cierre oral, limitación de movimientos, dolor periodontal y facial, cefaleas, hasta los de índole degenerati$\mathrm{va}(3)$.

Lo que indica que el debido diagnóstico de la afección por parte del prestador de servicio odontológico, permitirá de forma oportuna la implementación de un plan de tratamiento sea este la corrección de hábitos o la cirugía, para la restauración de la funcionalidad de la articulación.

Donde la eficacia del diagnóstico se hace necesaria; el odontólogo como parte de su formación académica, está preparado para interpretar y analizar los métodos más eficaces para el diagnóstico de ciertas patologías, en este caso de las TTM, diferentes estudios en Latinoamérica en la línea de investigación de Estomatología en específico de los TTM solo aplican un método para determinar la existencia o no de dichas afecciones, entre una de ellas el Test de Helkimo el cual es el método estándar para determinar TTM, otros emplean el Test de Krogh - Paulsen, Test de Screening o la escala Criterios de Diagnóstico para los Trastornos Temporomandibulares (DC / TTM)(4-8), entre ellos unos poseen dimensiones que los otros no consideran, ya que este tipo de TTM son tratados por causas multifactoriales, y el diagnostico inadecuado puede causarle al paciente un tratamiento inadecuado o prolongado.

Lo que hace preciso que se complementen y contrasten varios métodos para llegar a un diagnostico eficiente y eficaz, siguiendo la literatura actual la comparación entre dos test o escala permite lograr la efectividad del diagnóstico de los TTM $(8,9)$. Teniendo en cuenta estas ideas el presente estudio tuvo como objetivo evaluar la eficacia de los Test de Helkimo y Krogh - Paulsen para el diagnóstico de la disfunción temporomadibular, donde se estableció la capacidad diagnostica de cada test y las medidas de sensibilidad y especificidad.

\section{MÉTODOS Y MATERIALES}

A nivel mundial la mayor causa de la consulta odontológica se debe a la caries dental y a enfermedades periodontales, y solo cuando estas presentan algún grado de dolor o de impedimento para las actividades diarias del paciente, lo mismo ocurre cuando de afecciones sobre el aparato gnático se trata, aún cuando más de $70 \%$ de la población mundial (1) padezca de este tipo de afecciones, la principal causa de asistencia a la consulta odontológica es la presencia de dolor incapacitante en este sistema (2), este tipo de sintomatología presenta relativa importancia en la calidad de vida del individuo ya que se encarga de las acciones de la masticación, deglución, fonación y expresividad facial (1).

Este tipo de sintomatologías son clasificadas como disfunción o trastorno en la Articulación Temporomandibular (TTM), las cuales van desde ruidos en la articulación, dolores de los músculos masticadores, alteraciones en el cierre oral, limitación de movimientos, dolor periodontal y facial, cefaleas, hasta los de índole degenerati$\mathrm{va}(3)$. 
Lo que indica que el debido diagnóstico de la afección por parte del prestador de servicio odontológico, permitirá de forma oportuna la implementación de un plan de tratamiento sea este la corrección de hábitos o la cirugía, para la restauración de la funcionalidad de la articulación.

Donde la eficacia del diagnóstico se hace necesaria; el odontólogo como parte de su formación académica, está preparado para interpretar y analizar los métodos más eficaces para el diagnóstico de ciertas patologías, en este caso de las TTM, diferentes estudios en Latinoamérica en la línea de investigación de Estomatología en específico de los TTM solo aplican un método para determinar la existencia o no de dichas afecciones, entre una de ellas el Test de Helkimo el cual es el método estándar para determinar TTM, otros emplean el Test de Krogh - Paulsen, Test de Screening o la escala Criterios de Diagnóstico para los Trastornos Temporomandibulares (DC / TTM)(4-8), entre ellos unos poseen dimensiones que los otros no consideran, ya que este tipo de TTM son tratados por causas multifactoriales, y el diagnostico inadecuado puede causarle al paciente un tratamiento inadecuado o prolongado.

Lo que hace preciso que se complementen y contrasten varios métodos para llegar a un diagnostico eficiente y eficaz, siguiendo la literatura actual la comparación entre dos test o escala permite lograr la efectividad del diagnóstico de los TTM $(8,9)$. Teniendo en cuenta estas ideas el presente estudio tuvo como objetivo evaluar la eficacia de los Test de Helkimo y Krogh - Paulsen para el diagnóstico de la disfunción temporomadibular, donde se estableció la capacidad diagnostica de cada test y las medidas de sensibilidad y especificidad.

\section{Limitación en el rango del movimiento mandibular Apertura máxima:}

40 mm ó más: sin limitación o apertura normal' 30 a 39 mm: limitación leve

Menos de 30mm: limitación severa

\section{Máximo deslizamiento a la derecha:}

$7 \mathrm{~mm}$ o más: deslizamiento normal

4 a 6 mm: limitación leve del deslizamiento

0 a $3 \mathrm{~mm}$ : limitación severa del deslizamiento
(0 punto)

(1 punto)

(5 puntos)

Máximo deslizamiento a la izquierda: Será examinado de manera similar que el derecho.

\section{Máxima protrusión:}

$7 \mathrm{~mm}$ o más: movimiento protrusivo normal

4 - $6 \mathrm{~mm}$ : limitación leve del movimiento protrusivo

$0-3 \mathrm{~mm}$ : limitación severa del movimiento protrusivo

$$
\begin{aligned}
& \text { (0 punto) } \\
& \text { (1 punto) } \\
& \text { (5 puntos) }
\end{aligned}
$$

\section{Índice de movimiento}
a. Movilidad normal:
0 punto
b. Moderado deterioro de la movilidad:
1 - 4 puntos
c. Grave deterioro de la movilidad:
5 - 20 puntos

Se da un valor de 0 para a, 1 para b, 5 para c, en dependencia del grado de limitación del movimiento. 


\section{Función de la ATM}

-Apertura y cierre con desviación mandibular de $2 \mathrm{~mm}$ en apertura o cierre sin ruidos.

(0 punto)

-Sonidos articulares o desviación mandibular

el movimiento de apertura, o ambas cosas.

(1 punto)

- Traba o luxación, con sonido o sin él.

(5 puntos)

\section{Estado muscular}

Sin sensibilidad a la palpación/manipulación funcional

(0 punto)

Sensibilidad a la palpación/manipulación funcional en 3 sitios

(1 punto)

Sensibilidad a la palpación/manipulación funcional en más de 3 sitios

\section{Estado de la ATM}

-Sin dolor espontáneo ni a la palpación

(0 punto)

-Dolor a la palpación periauricular unilateral o

bilateral de la articulación

(1 punto)

-Dolor a la palpación vía conducto auditivo

externo y periauricular

(5 puntos)

Dolor al movimiento mandibular

Movimiento mandibular sin dolor:

(0 punto)

Dolor referido a un solo movimiento:

(1 punto)

Dolor referido a dos o más movimientos:

(5 puntos)

Tabla N 1 . Clasificación de gravedad grado de TTM, según Helkimo

\begin{tabular}{|c|c|}
\hline Indice Anamnés ico & DTM \\
\hline 0 & SIN TTM \\
$1-4$ & LEVE \\
$5-9$ & MODERADO \\
$10-14$ & SEVERA GRADO I \\
$15-19$ & SEVERA GRADO ॥ \\
$20-25$ & SEVERA GRADO II \\
\hline
\end{tabular}

Para el segundo instrumento el Test de Krogh - Paulse se realizó un examen de los músculos y la articulación por medio de la palpación y la manipulación funcional de los músculos temporal, masetero, pterigoideo medial, pterigoideo lateral, el esternocleidomastoideo y los cervicales posteriores del cuello. Respondiendo a los siguientes indicadores 
Tabla N². Indicadores del Test de Krogh - Paulse

\begin{tabular}{|c|c|c|}
\hline Signos y sintomas & Si & No \\
\hline \multicolumn{3}{|l|}{1 A bertura menor a $40 \mathrm{~mm}$} \\
\hline \multicolumn{3}{|l|}{2 Irregularidades en movimiento de abertura y cierre } \\
\hline \multicolumn{3}{|l|}{3 Dolor muscular a la palpación } \\
\hline \multicolumn{3}{|l|}{4 Dolor en A TM } \\
\hline \multicolumn{3}{|l|}{5 Chasquido o crujido } \\
\hline \multicolumn{3}{|l|}{6 Traba en apertura y cierre } \\
\hline \multicolumn{3}{|l|}{$\begin{array}{l}7 \text { Inestabilidad entre Relación Céntrica y Posición de máxima } \\
\text { intercuspidación }\end{array}$} \\
\hline \multicolumn{3}{|l|}{8 Deslizamiento anterior $>1 \mathrm{~mm}$} \\
\hline 9 Deslizamiento Lateral & & \\
\hline
\end{tabular}

Se analizan según estos criterios:

$\begin{array}{lll}\text { Sano } & & \text { Ningún aspecto positivo. } \\ \text { Perturbación } & \text { Un aspecto positivo. } \\ \text { Riesgo } & \text { Dos aspectos positivos. } \\ \text { Disfunción } & \text { Tres aspectos positivos. } \\ \text { Ítems } 6 \text { o } 9 \text { positivos (son determinantes) }\end{array}$

Los datos fueron organizados en Microsoft Excel tomando en cuenta la dicotomización de los datos en valores negativos y positivos para TTM, posteriormente analizados por el Software SPSS (versión 24?XXXX) utilizando la estadística descriptiva para el cálculo de las frecuencias y porcentajes. En cuanto al cálculo de la capacidad diagnostica se realizó la prueba Chi2, con un nivel de significancia de $\mathrm{p}<0.05$ para ser considerado estadísticamente significativo.

\section{RESULTADOS}

Realizando la evaluación entre ambos Test se determina que el Test de Helkimo en comparación con el Test Krogh - Paulsen es 16,6\% superior para el diagnóstico positivo de TTM, pero mediante la prueba $C h i 2=3,268$ con un $\mathrm{p}=0,0706$ no se muestran diferencias estadísticamente significativas. 
Tabla $N^{\circ}$ 3. Comparación entre resultados de afección de TTM utilizando el test de Helkimo y Krogh - Paulsen

\begin{tabular}{|c|c|c|c|c|c|c|}
\hline \multirow{3}{*}{ Valor de prueba } & \multicolumn{4}{|c|}{ Test Diagnóstico } & \multirow{2}{*}{\multicolumn{2}{|c|}{ TOTAL }} \\
\hline & \multicolumn{2}{|c|}{ Helkimo } & \multicolumn{2}{|c|}{ Krogh - Paulsen } & & \\
\hline & $\mathrm{N}$ & $\%$ & $\mathrm{~N}$ & $\%$ & $\mathrm{~N}$ & $\%$ \\
\hline NEGATIVA & 2 & 6.7 & 7 & 23.3 & 9 & 15.0 \\
\hline POSITIVA & 28 & 93.3 & 23 & 76.7 & 51 & 85.0 \\
\hline TOTAL & 30 & 100.0 & 30 & 100.0 & 60 & 100.0 \\
\hline
\end{tabular}

Tabla N${ }^{\circ}$. Sensibilidad y Especificidad para el Test de Helkimo y Krogh - Paulsen

\begin{tabular}{lccc}
\hline \multirow{2}{*}{$\begin{array}{l}\text { Test de Krogh } \\
\text { Paulsen }\end{array}$} & \multicolumn{2}{c}{ Test de Helkimo } & Total \\
\cline { 2 - 3 } & Enfermos & Sanos & 23 \\
\hline Positivos & 22 & 1 & 7 \\
negativos & 6 & 1 & 30
\end{tabular}

En referencia al Test de Helkimo se determinó para la sensibilidad del Test de Krogh - Paulsen que esta es de $78.6 \%$ y la especificidad de 50\%. (FALTA LA PRUEBA POR LA CUAL SE CALCULO LA SENSIBILIDAD Y ESPECIFICIDAD)

\section{DISCUSIÓN}

Dentro de la labor del dentista la constante capacitación proveer un factor de éxito en el establecimiento de diagnósticos acordes a la sintomatología del paciente. La preocupación incide en la formación curricular de las actuales universidades peruanas, donde los estudiantes no tienen un área específica de estudio, por tanto para abarcar los complementos del plan de estudio, la revisión teórica se hace necesaria.

Entre ellas anatomía, ortodoncia, rehabilitación, entre otras; pero esto no prepara a clínico a las posibles particularidades de la atención del paciente. Aun cuando se evidencie que más del $70 \%$ de los pacientes que acuden a una consulta odontológica tienen la presencia de por lo menos un signo de disfunción (ruido, dificultad de apertura) lo cual atribuye la importancia para el diagnóstico eficaz de dicha afección. Y de esta forma, la preocupación incita a la revisión constante de la literatura y de encontrar medios confiables para el diagnóstico. De acuerdo a la búsqueda se seleccionó los Test de Helkimo y Krogh - Paulsen como instrumentos de eficacia comprobada a nivel mundial, teniendo el primero una vigencia de cincuenta años desde su elaboración; aun cuando existen muchos test y escalas creados para tal fin, la escogencia de estos dos se debió: uno a que el primero presenta alta fiabilidad en el diagnóstico y el segundo está siendo aplicado en la actualidad por la estructura de sus dimensiones; ya que se determina que los TTM son multifactoriales y de diferentes causas.

De acuerdo a (8)la comparación de diferentes instrumentos para la detección de un TTM permite una visión teórica y metodológica para diagnóstico de dolencia hacia esta articulación. Esto permitirá un desarrollo y mejora de las líneas de investigación del área odontológica para el desarrollo de instrumentos coherentes, aplicables, consistentes y fiables en la identificación de patologías y en la implementación de planes de tratamiento más congruentes con el avance tec- 
nológico que esta área clínica ha presentado en las últimas décadas en cuanto al diagnóstico y la terapia. Es entonces que las diversas metodologías aplicadas en los instrumentos existentes hace difícil una comparación en cuanto a la aplicabilidad y valoración, debido a que unos acceden a la fase clínica anamnésica y otros a la signología diagnóstica, hace factible que se contemple una reestructuración de los mismos.

En específico para los teste seleccionados el de Helkimo es rico en contenido analítico, pues considera tres componentes mínimos para ser aplicado: el componente muscular, aspectos de oclusión o relación intermaxilar y sintomatología de la Articulación Temporomandibular, lo cual le adquiere una capacidad diagnostica de mayor precisión(10). De acuerdo a los criterios para ambos test se encontró que 43,3\% de los pacientes presentan un TTM leve, y para la categoría TTM moderado este porcentaje se repite, y un $6,7 \%$ con severo Grado 1 de TTM, haciendo la comparación con el Test de Krogh - Paulsen se determinó que $76,7 \%$ de los sujetos tienen una Disfunción Temporomadibular. Lo cual haciendo una agrupación entre los sujetos sanos y enfermos los porcentajes de coincidencia con el diagnósticos fue parecido, pero es de hacer notar que el Test de Helkimo presenta un 93,3\% (Tabla 3) de diagnósticos positivos para TTM.

Aun cuando no hay diferencias significativas para determinar cuál test es más adecuado, se toma en consideración los datos de (10) en cuanto a la estructuración del Test de Krogh - Paulsen sobre el instrumento más adecuado para diagnosticar a sujetos sanos de TTM, así mismo se enfatiza que debido a su estructura es adecuado a ser aplicado en poblaciones de niños en edades escolares(11).

En el caso del Test e Helkimo cuando es comparado con otro instrumento como el Test Screenig(8) este presenta mayor pretendencia de TTM en más de un $10 \%$. Y atribuyéndole su capacidad analítica permite evaluar anamnésica y clínicamente a los sujetos en contraste al test Screening. Es de esta forma, se estableció por medio de prue- bas estadísticas que no existen diferencias significativas entre ambos Test, por lo que la presente investigación realiza una contribución al análisis de la consistencia del Test de Krogh - Paulse en referencia al Test de Helkimo, los resultados en cuanto a la sensibilidad concuerdan con (10), aun cuando discrepan los valores en cuanto a la especificidad, siendo el de este estudio de $50 \%$ y del autor precitado de $100 \%$, lo cual señala diferencias en cuanto a los criterios metodológicos.

Entonces, la reiterada evaluación permite su permanencia para el diagnóstico eficaz de los TTM, aun cuando no se debe obviar la comparación entre varios instrumentos para lograr un diagnóstico adecuado en cuanto a la edad (10) y a las actividades psicosociales y los trastornos clínicos $(7,9)$.

En vista de la variedad de instrumentos empleados para determinar la Difusión Temporomandibular, se exhorta al análisis de la vigencia de dichos Test y escalas, donde estos presenten concordancia, coherencia, fiabilidad y consistencia, para de esta forma contribuir a la mejora de la línea de investigación de TTM.

\section{CONCLUSIONES}

El presente estudio concluye que el análisis de las afecciones Temporomandibulares debe efectuarse entre varios instrumentos (test o escalas) que permitan el diagnostico eficaz entre los cuales se demuestren consistencia, coherencia y concordancia en su aplicabilidad. El Test de Helmiko y el Test Krogh - Paulsen no presentan diferencias en su capacidad de diagnóstico. Así mismo para el análisis de la especificidad del Test de Krogh - Paulsen se deben establecer parámetros metodológicos a nivel mundial, para que no exista discrepancia entre las interpretaciones.

\section{REFERENCIAS}

1. Quiroz P, Monje F, Vázquez E. Diagnóstico de la patología de la articulación temporomandibular [Internet]. Protocolos clínicos de la Sociedad Española de Cirugía Oral y Maxilofacial; 2014. Disponible en: http://www.secom.org/ wp-content/uploads/2014/01/cap19.pdf 
2. Tirado L. Trastornos temporomandibulares: algunas consideraciones de su etiología y diagnóstico. Rev Nac Odontol [Internet]. 16 de mayo de 2015;11. Disponible en: https://www.researchgate.net/publication/277907226

3. Lescas O, Hernández M, Sosa A, Sánchez M, Ugalde C, Ubaldo L, et al. Trastornos temporomandibulares. Rev Fac Med UNAM. 2011;55(1):8.

4. Espinoza V. Evaluación Clínica de trastornos Temporomandibulares en pacientes de 18 a 35 años atendidos en la Clínica Odontológica de la Universidad de la Américas en el periodo 2015-2016 [Internet] [para optar al Título de Odontóloga]. [Ecuador]: Universidad de Las Américas; 2016. Disponible en: http://dspace.udla.edu.ec/bitstream/33000/5431/1/UDLA-EC-TOD-2016-42.pdf

5. Montero J, Semykina O, Morais L. Trastornos temporomandibulares y su interacción con la postura corporal. Rev Cuba Estomatol. marzo de 2014;51(1):3-14.

6. Vásconez M, Bravo W, Villavicencio E. Factores asociados a los trastornos temporomandibulares en adultos de Cuenca, Ecuador. Rev Estomatológica Hered. 28 de junio de 2017;27(1):5.

7. Anaya S, Casanova C, Barrera J, Benavides R, Sánchez N, Buriticá A. Prevalencia de trastornos de la articulación temporomandibular según los criterios diagnósticos para la investigación en pacientes preortodónticos. Universitas Odontologica. 2014;33(71):19-28.

8. Aquino L. Estudio comparativo entre el Índice de Helkimo y el Test de Screening en el diagnóstico de Trastornos Temporomandibulares en estudiantes de quinto grado de la IES Politécnico Regional de Los Andes, Juliaca, 2015 [Internet] [para optar al Título de Cirujano Dentista]. [Puno]: Universidad Nacional de Altiplano; 2015 [citado 2 de junio de 2019]. Disponible en: http://repositorio.unap.edu.pe/ bitstream/handle/UNAP/1786/Aquino_Apaza_Luz_Marina.pdf? sequence $=1$ \&isAllowed $=y$

9. Flores M del C. Estudio comparativo del índice de criterios diagnósticos de los trastornos temporomandibulares y el índice de Helkimo en una población de estudiantes de odontología en Sinaloa, México [Tesis Doctoral]. [Granada]: Universidad de Granada; 2008.

10. Conerjo J. Sensibilidad Y Especificidad del Indice de Krogh Poulsen en el diagnóstico de los Trastornos Temporomandibulares. Revista de Investigación UNMSM [Internet]. 2014;1(3). Disponible en: http://revistasinvestigacion. unmsm.edu.pe/index.php/odont/article/view/3428

11. Verdugo L, García R, Castro A. Disfunción de la Articulación Temporomadibular (ATM) en pacientes de nuevo ingreso a la clínica de Ortodoncia y Ortopedia de la Uni- versidad Autonóma de Sinaloa. Revista Medica de la UAS. 2010;1(2):7-11. 